\title{
Características químicas dos resíduos de serragem segregados de rochas ornamentais do estado do Espírito Santo
}

\author{
José Júlio Garcia de Freitas ${ }^{1 *}$, Valério Raymundo ${ }^{2}$, Honério Coutinho de Jesus ${ }^{3}$
}

\begin{abstract}
Resumo Embora existam vários trabalhos que sugiram a utilização de resíduos do beneficiamento de rochas ornamentais para fabricação de telhas, tijolos, ladrilhos etc., de ampla aplicação na Construção Civil, nenhum deles até o presente momento apresentou, de forma satisfatória e sistemática, estudos de caracterização química dessa lama residual. Esses resíduos industriais de rochas ornamentais são destaques devido ao enorme impacto ambiental e visual gerados, pois grandes volumes desse material são gerados diariamente na serragem dos blocos rochosos. No gerenciamento de resíduos, a caracterização é considerada a etapa mais importante e que norteia atitudes a serem tomadas na destinação dos mesmos. O presente trabalho buscou o fornecimento de subsídios para caracterização química inorgânica dos resíduos de serragem do setor de rochas ornamentais, oriundas do estado do Espírito Santo, partindo de uma amostragem segregada, por tipo comercial de rocha, que consistiu na coleta de amostras diretamente no descarte dos teares de serragem, após a passagem da lama por um hidrociclone, e, portanto, antes de serem misturadas no tanque de efluentes. Para cada amostra coletada, foram obtidos em laboratório os valores de $\mathrm{pH}$, teor de umidade, teor de sólidos secos e teores de metais de interesse ambiental. Na determinação dos teores metálicos, foram utilizadas as técnicas analíticas de técnicas instrumentais analíticas por absorção atômica (AAS, F AAS e GF AAS), as quais indicaram boas correlações entre teores de Fe e Ca total e entre $\mathrm{Cr}$ total e pH original. Além disso, elevados valores de $\mathrm{pH}$, resultantes do excesso de cal empregado na produção industrial, foram detectados para alguns resíduos analisados, revelando, para alguns materiais, uma classificação do tipo "Classe I" - resíduos perigosos. O teor de umidade médio de 44,3\%, com 13\% de desvio padrão, indica que a utilização da água do setor está longe de ser sustentável, mas parece haver uniformidade nesse consumo. O presente trabalho deixa como sugestão, ao processo de serragem de blocos de rochas ornamentais, em função dos resultados obtidos, que passe a monitorar resíduos industriais quanto aos parâmetros $\mathrm{pH}$ e teores de metais tóxicos periodicamente.
\end{abstract}

Palavras-chave: resíduos sólidos; caracterização; rochas ornamentais.

Abstract Chemical characteristics of sawing waste segregated of ornamental stones from Espírito Santo state. Although there are several studies suggesting the use of ornamental rocks tailing for the manufacture of tiles, bricks, tiles, among others, of large application in Construction, none of them until this moment has presented satisfactory and systematic studies of sludge residual chemical characterization. Such industrial waste of ornamental stones is highlighted due to the enormous environmental and visual impacts generated, because large volumes of material are generated daily in the sawdust of stone blocks. For the management of waste, the characterization is considered the most important stage and the one that guides attitudes to be taken in assigning the same. This work sought the provision of subsidies for the inorganic chemistry characterization of the sawing waste sector from ornamental stones from the Espírito Santo state, starting from a sampling segregated, commercial type of rock, which consisted in the collection of samples directly in the discarding of handlooms sawdust, after the passage of sludge by a hydrocyclone and, therefore, before being mixed in the effluent tank. For each collected sample, in the laboratory, $\mathrm{pH}$ values, moisture content, humid and dried solid contents, and metal content of environmental interest were obtained. In the determination of metal levels, AAS, F AAS and GF AAS analytical techniques were used, which indicated good correlations between $\mathrm{Fe}$ and total $\mathrm{Ca}$ and between total $\mathrm{Cr}$ and original $\mathrm{pH}$. Furthermore, high $\mathrm{pH}$ values, resulting from the excess of lime used in industrial production, were detected for some wastes, showing, for some materials, a type classification of «Class I» - hazardous waste. The average moisture content of $44.3 \%$, with $13 \%$ standard deviation, indicates that using water in the industry must be sustained further, but this appears to be a uniform consumption. This paper has suggested, as to the process of sawing blocks of stone, according to the obtained results, that it starts periodically monitoring industrial wastes for the $\mathrm{pH}$ and toxic metals levels parameters.

Keywords: solid waste; characterization; ornamental stones.

${ }^{1}$ Departamento de Química, Programa de Pós-Graduação em Química, Universidade Federal do Espírito Santo - UFES, Vitória (ES); Universidade Vila Velha - UVV, Vila Velha (ES), Brasil. E-mail: julio.garcia@uvv.br, profjuliogarcia@hotmail.com

${ }^{2}$ Centro de Ciências Agrárias, Programa de Pós-Graduação em Produção Vegetal, Universidade Federal do Espírito Santo - UFES, Alegre (ES), Brasil. E-mail: valerio30@gmail.com

${ }^{3}$ Departamento de Química, Laboratório de Química Analítica - LQA, Universidade Federal do Espírito Santo - UFES, Alegre (ES), Brasil. E-mail: honerio2@cce.ufes.br

*Autor correspondente 
INTRODUÇÃO A indústria do beneficiamento de rochas ornamentais já mostrou ao estado do Espírito Santo seu grande potencial produtivo e sua grande capacidade de gerar divisas. Contudo, há carências no setor que necessitam de investimentos na área humana, no melhoramento de processos, na saúde ocupacional ou na área ambiental. O setor carece de pesquisas que fomentem novas tecnologias produtivas e que viabilizem, pelo menos, a qualidade de vida aos assalariados que vivem do setor e das populações de entorno das unidades de produção.

Durante o beneficiamento dos blocos de granito nos teares que utilizam granalha (ferro/aço pulverizados) como material abrasivo, são produzidos finos de ferro ou aço em concentração com minerais presentes nas rochas serradas, em função do atrito que ocorre entre as lâminas de aço, a mistura abrasiva e o bloco rochoso. Esses finos provocam alterações nas condições reológicas da mistura abrasiva diminuindo a produtividade. Assim, em intervalos previamente calculados em função da metragem horária cortada pelo tear, são realizados expurgos (retiradas) da mistura abrasiva em ciclones deslamadores, buscando um corte na fração inferior a $0,42 \mathrm{~mm}$ para eliminação dos finos (pó de rocha + granalha não ativa + cal hidratada + $\mathrm{H} 2 \mathrm{O})$ do processo.

Normalmente, esses resíduos são descartados em bacias de deposição adjacentes à serraria (Vieira Júnior 2001). Este material apresenta em torno de $40 \%$ em massa de água e $\mathrm{pH}$ em torno de 10 a 12,5.

Para uma tentativa de aproveitamento destes rejeitos, faz-se necessário um estudo das características químicas e mineralógicas, das possibilidades de segregação dos resíduos e da demanda mercadológica industrial.

Os ensaios de solubilização foram aplicados, gerando-se um instrumento de avaliação da interação entre resíduos sólidos expostos ao meio aquoso desprovido de outras espécies químicas senão às presentes nas amostras utilizadas, simulando, assim, a exposição de resíduo a chuvas. Portanto, foi possível ter uma ideia do determinado impacto químico a ser causado ao ambiente quando o referido resíduo é disposto sem que haja nenhum tipo de gerenciamento ou tratamento prévio do mesmo, como ocorre normalmente na maioria das empresas do setor de rochas ornamentais do Espírito Santo (ES).

O estudo proposto está relacionado com a “caracterização química inorgânica da lama residual liberada após corte ou serragem de blocos de granitos e mármores de forma segregada", possibilitando algum subsídio técnico-científico para a gestão adequada desse rejeito. Neste primeiro momento foram conduzidas análises físico-químicas como avaliação do pH original do resíduo, determinação do teor de sólidos úmidos e secos $\left(42{ }^{\circ} \mathrm{C}\right)$ e teores totais de metais de interesses químico e ambiental.

MATERIAIS E MÉTODOS As amostragens foram realizadas em distritos de Cachoeiro de Itapemirim, nas localidades de Gironda, Vargem Alta de Soturno e Atílio Vivácqua, com a colaboração de diversas empresas filiadas ao Sindicato das Indústrias de Rochas Ornamentais, Cal e Calcário do Estado do Espírito Santo (Sindirochas). As amostras foram levadas ao Laboratório de Química Analítica da Universidade Federal do Espírito Santo (UFES) para avaliações dos parâmetros físico-químicos e analíticos, os quais eram preconizados na norma de classificação de resíduos sólidos da Associação Brasileira de Normas e Técnicas (ABNT) (NBR 10.004/ 2004a), em caixas de isopor, em veículo particular, em uma viagem de aproximadamente duas horas.

Após a lâmina de corte ser totalmente coberta no corte, incrementos de lama residual foram coletados, num total de dez (Fig. 1), cada qual com volume aproximado de 1,5 L, após a passagem pelo hidrociclone no poço principal (Fig. 2). Foram extraídos e recolhidos em um balde plástico específico por tipo comercial de rocha de aproximadamente $15 \mathrm{~L}$. Esse mesmo procedimento foi repetido até se chegar a amostras do início, meio e fim da serragem do bloco rochoso.

Para compor cada uma dessas amostras, para os diferentes tipos comerciais de rochas ornamentais estudados, após a etapa de preparo no balde de $15 \mathrm{~L}$, realizou-se a homogeneização e o quarteamento, gerando-se quatro amostras representativas, de 1 L cada (Fig. 1), em relação ao processo de corte de rochas, sendo uma amostra titular (amostra final) e outras três de arquivo 
Universo ou lote

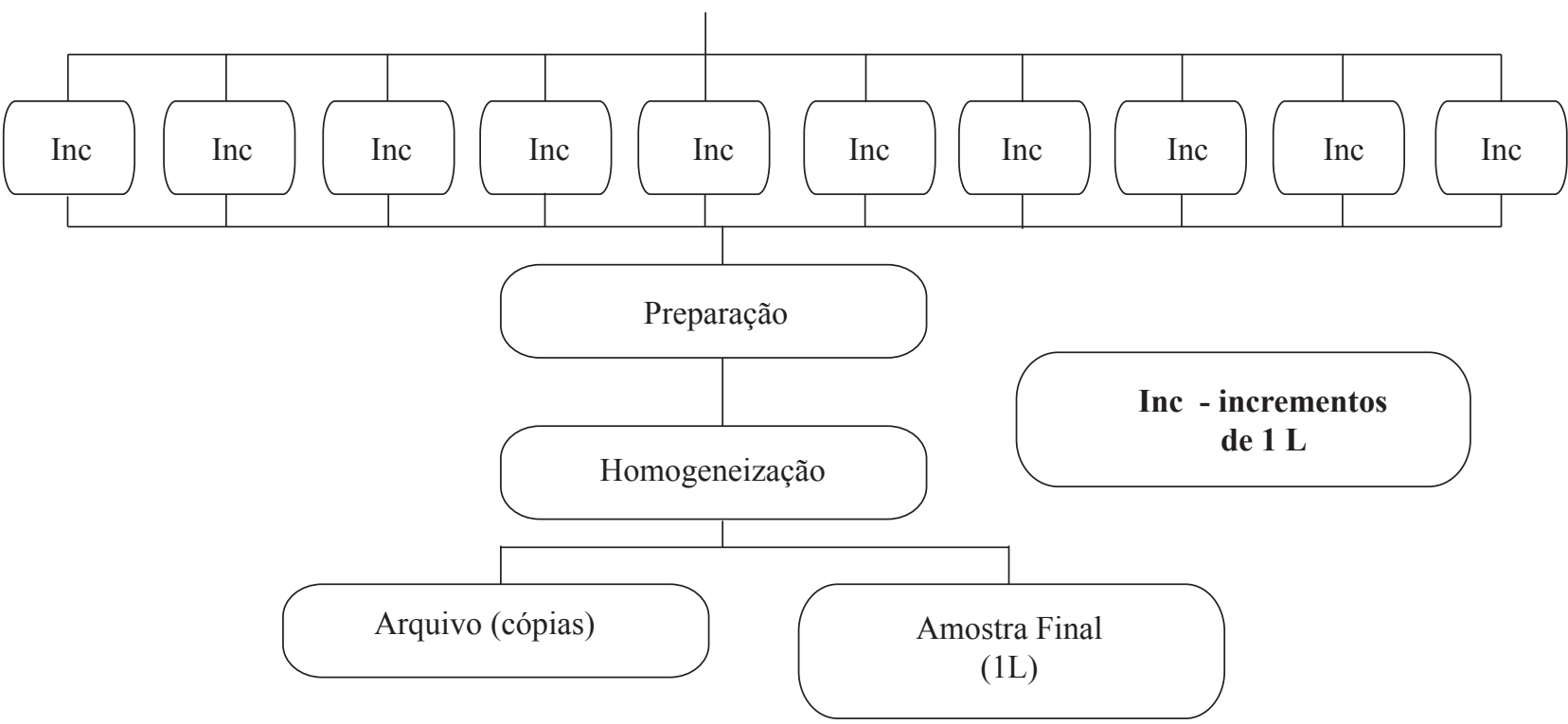

Figura 1 - Diagrama mostrando a metodologia adotada na amostragem de resíduos sólidos de corte de granitos e mármores. Formatação adaptada de Sampaio et al. 2007.

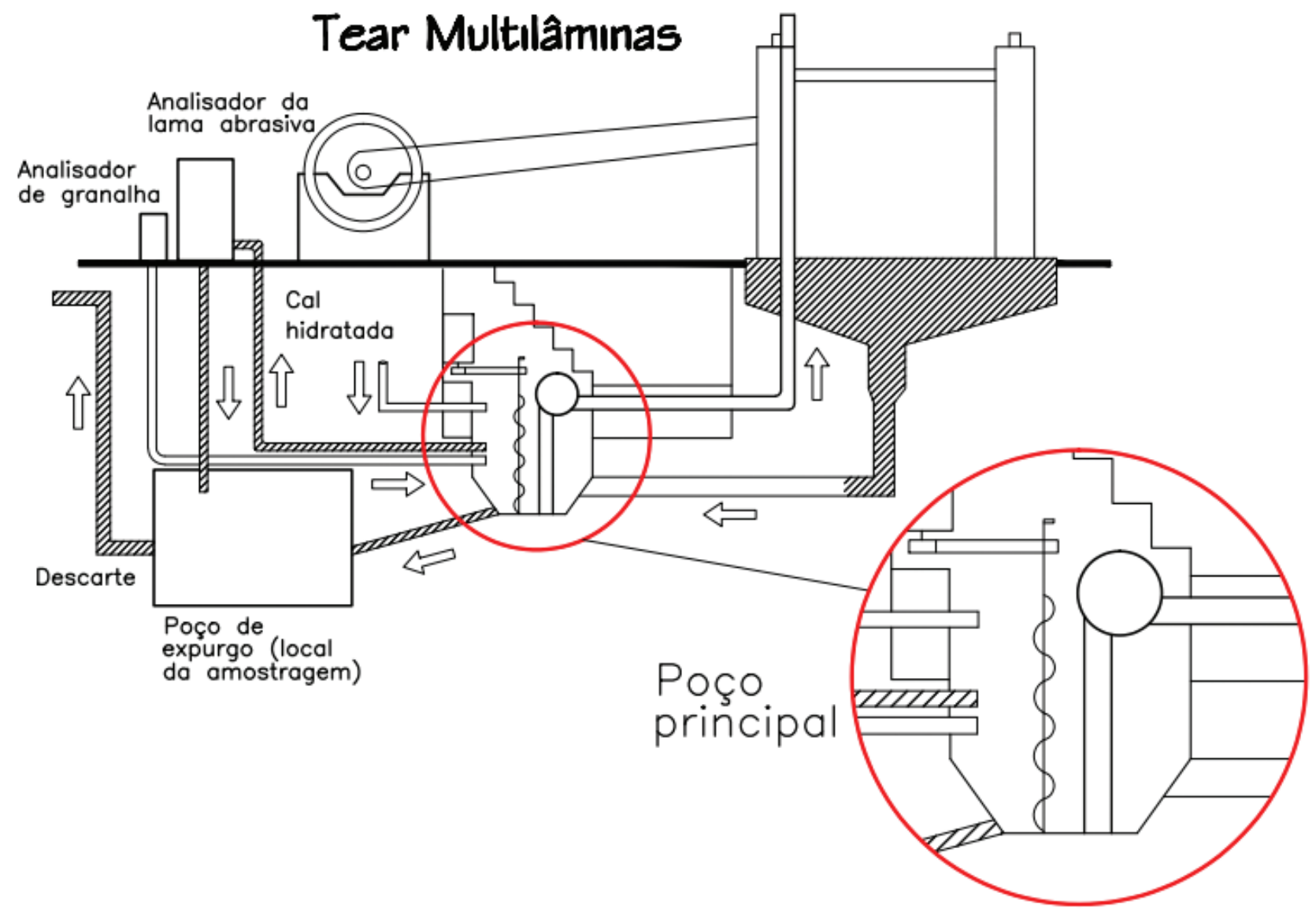

Figura 2 - Esquema modelo de um tear multilâminas, com explicitação do local em que as amostragens foram realizadas na área dentro do círculo em vermelho. Fonte: Peyneau \& Pereira 2004. 
(arquivo - cópias), ou seja, reservas para qualquer imprevisto.

As amostras coletadas passaram por um estudo, por meio de uma sequência de ensaios que visam à caracterização físico-química das mesmas (determinação do pH in situ e no laboratório, temperatura e umidade relativas a $42{ }^{\circ} \mathrm{C}$ ), de acordo com as normas para caracterização de resíduos sólidos da ABNT (NBR 10.004 2004a).

\section{Avaliação do pH Imediatamente após a re-} cepção das amostras residuais em laboratório, estas passaram por um monitoramento de alcalinidade, por meio da utilização de um medidor de pH, modelo PM608, da marca ANALON.

Apesar de ser uma análise físico-química preliminar, o monitoramento do $\mathrm{pH}$ das amostras originais verificou valores fora da faixa ( 2 a 12,5), sendo suficiente para considerar o resíduo perigoso (NBR 10.004 2004a).

\section{Ensaio de solubilização para resíduos sóli-}

dos Cada amostra de resíduo foi seca a 42 ${ }^{\circ} \mathrm{C}$, em bandejas plásticas, Plastigel de $3.000 \mathrm{~mL}$, em estufa com circulação forçada de ar, modelo Q314M-242, da marca CALLMEX (Florianópolis, Santa Catarina). No caso do rejeito em questão, geralmente, foram necessárias 72 horas, em média, nessas condições, para que o material fosse seco. O processo de secagem foi monitorado em balança semianalítica, modelo MARK 2200 de marca BEL Engineering (Monza, Itália), retirando-se as bandejas plásticas reiteradas vezes até que a constância de massa fosse verificada.

Foram determinados os teores de umidade (Tab. 1) conforme norma supracitada, as amostras foram trituradas até se obter granulometria de 9,5 mm, verificadas em peneira própria; pesaram-se alíquotas em duplicata de cada material residual de aproximadamente $250,0 \mathrm{~g}$ em balança de precisão, em béqueres de $1,5 \mathrm{~L}$ ou em recipientes plásticos de forma e volumes similares. Esse ensaio (solubilização) submeteu os resíduos secos a superfícies similares de contato com a água de osmose reversa $(1,0$ L), em agitação regular, de cinco minutos, em agitador rotatório, modelo MA 266E, da marca Marconi (Campinas, São Paulo).

Após tal procedimento, os recipientes foram selados com filme delgado de PVC, para evitar possíveis contaminações do analito, e foram repousados por sete dias corridos. Vencidos os prazos de repouso, as amostras foram desseladas e filtradas, em aparelho de filtragem a vácuo, guarnecido com membrana filtrante de fibra de vidro de $0,45 \mu \mathrm{m}$ de porosidade, obtendo-se o extrato solubilizado. Após a obtenção, os extratos solubilizados passaram por uma estabilização ácida, resultando em extratos acidificados de $\mathrm{pH}$ abaixo de 2,0, evitando-se, assim, a formação de complexos metálicos, os quais poderiam inviabilizar análises químicas instrumentais posteriores.

\section{Dissolução total das amostras (dissolução total} de sólidos) Aproximadamente $500 \mathrm{mg}$ das amostras secas em estufa $\left(42{ }^{\circ} \mathrm{C}\right)$ foram transferidas para béqueres de Teflon $^{\odot}$ e digeridas com as seguintes misturas, sequencialmente, em chapa elétrica aquecida a $250{ }^{\circ} \mathrm{C}$, contida num sistema de exaustão. Cada etapa era levada até quase a secura. O resíduo final foi dissolvido com $\mathrm{HNO} 3$ $0,2 \%$ e o volume completado para $50 \mathrm{~mL}$ em tubos de polipropileno, após filtração das soluções com papel de filtro quantitativo, como pode ser observado a seguir:

- Primeira etapa: $10 \mathrm{~mL}$ de $\mathrm{HNO}_{3}+5 \mathrm{~mL}$ de HF;

- Segunda etapa: $5 \mathrm{~mL}$ de $\mathrm{HNO}_{3}+5 \mathrm{~mL}$ de HF;

- Terceira etapa: $5 \mathrm{~mL}$ de $\mathrm{HNO}_{3}+5 \mathrm{~mL}$ de $\mathrm{HClO}_{4}$;

- Quarta etapa: $5 \mathrm{~mL}$ de $\mathrm{HNO}_{3} \rightarrow$ no caso de ausência de resíduo, adição final de $5 \mathrm{~mL}$ de $\mathrm{H}_{2} \mathrm{O}_{2}$; e, no caso de presença de resíduo, adição de mais $5 \mathrm{~mL}$ de $\mathrm{HF}$, com posterior adição de $5 \mathrm{~mL} \mathrm{HNO}_{3}$ e $5 \mathrm{~mL}$ de $\mathrm{H}_{2} \mathrm{O}_{2}$.

O resultado obtido nessa etapa foi a completa dissolução da amostra seca, obtendo-se, assim, os possíveis metais de interesse ambiental presentes, plenamente solubilizados. Tais teores são importantes parâmetros químicos na correlação dos mesmos com o pH das amostras originais (Fig. 2).

Determinação de metais por técnicas de espectrometria de absorção atômica As soluções obtidas após os procedimentos de solubilização, como também de rejeitos digeridos em 
Tabela 1 - Teores de água obtidos após secagem dos resíduos a $42{ }^{\circ} \mathrm{C}$ para teste de solubilização (espaços sem dados: dados ainda não levantados)

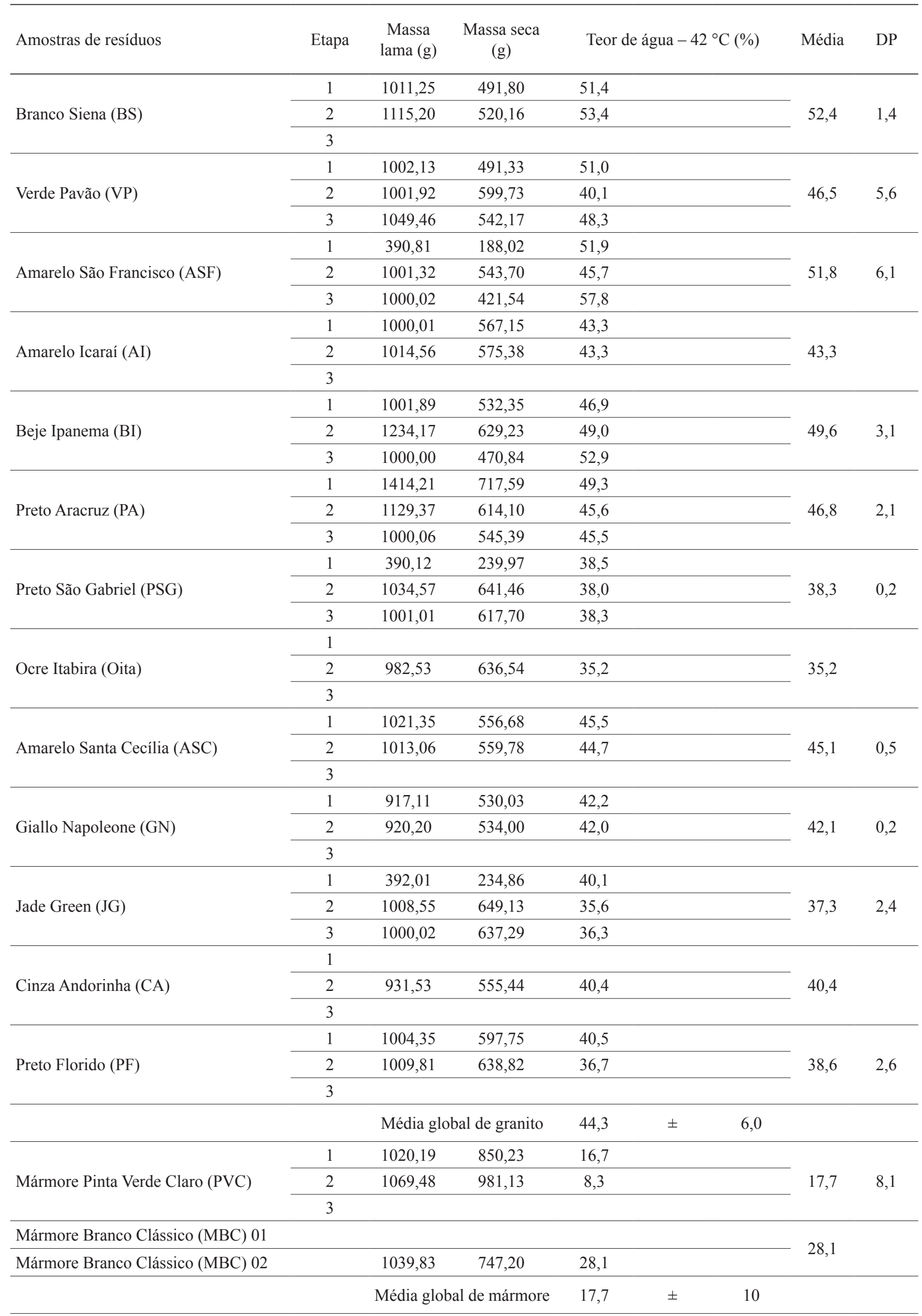


sua totalidade, foram analisadas pela técnica de espectrometria de absorção atômica (AAS) utilizando as condições padrões otimizadas e o corretor de fundo com lâmpada de deutério. Os metais ( $\mathrm{Fe}, \mathrm{Ca}$ e Al), como pode ser observado na Tab. 2 , foram determinados pela técnica de espectrometria de absorção atômica por chama (F AAS), modelo AA1275, da Varian. O metal Cr (Tab. 2) foi quantificado pela técnica de espectrometria de absorção atômica com forno de grafite (GF AAS), modelo AAS-5 da ZEISS, utilizando-se o modificador universal nitrato de paládio/nitrato de magnésio e tubo de grafite com aquecimento transversal (menor absorção de fundo). Na técnica de chama, $\mathrm{Al}, \mathrm{Cr}$ e $\mathrm{Ca}$ foram determinados com o uso da chama de óxido nitroso mais o acetileno. A utilização de duas diferentes técnicas analíticas para análise e determinação desses diversos metais foi realizada em função dos limites de detecção (Tab. 2) e das concentrações esperadas de cada uma das espécies químicas (previstas na NBR 10.004 da ABNT e em laudos técnicos realizados pelo LQA-UFES em materiais similares anteriores a presente pesquisa).

Foram obtidas as curvas analíticas com coeficientes de correlação satisfatórios $(r>0,999)$ e as condições otimizadas de trabalho.
Os limites de detecção, apresentados na Tab. 2, foram previamente levantados para cada um dos metais determinados, considerando-se igual a três vezes o desvio estimado Sy/x e dividido pela sensibilidade da reta a partir daquela de regressão obtida das curvas de calibração, em ambas as técnicas (SEMACE 2005).

\section{RESULTADOS E DISCUSSÃO}

Teor de água nas amostras Na Tab. 1 são apresentados os teores de água obtidos para as amostras de rejeitos após secagem a $42{ }^{\circ} \mathrm{C}$ até peso constante (NBR 10006 2004b), organizados por empresas.

A média global para granito foi de $44,3 \%$ $(\mathrm{n}=30)$ com desvio padrão relativo (RSD) de $13 \%$, o que aponta para um processo industrial de serragem bem uniforme entre as empresas, no que tange a quantidade de água utilizada na serragem dos blocos de granito. A média para mármore foi de $17,7 \%(n=3)$, típico para o processo de serragem das rochas macias, dispensando ainda grande parte da cal e granalha utilizada no corte de granitos, em teares convencionais. Desta forma, a lama gerada no corte de mármores é mais concentrada e o processo de corte é mais rápido e de menor

Tabela 2 - Teores totais de metais e pH das amostras originais de rejeito

\begin{tabular}{|c|c|c|c|c|c|c|}
\hline Amostra & Etapa & $\% \mathrm{Al}$ & $\% \mathrm{Fe}$ & $\% \mathrm{Cr}$ & $\% \mathrm{Ca}$ & $\mathrm{pH}$ original \\
\hline BS & 2 & 4,4 & 3,3 & 0,007 & 1,9 & 12,46 \\
\hline VP & 2 & 6,5 & 6,7 & 0,008 & 3,3 & 12,08 \\
\hline ASF & 1 & 4,9 & 10,0 & 0,054 & 4,7 & 12,80 \\
\hline AI & 2 & 5,1 & 5,6 & 0,035 & 2,6 & 12,91 \\
\hline BI & 2 & 4,2 & 6,0 & 0,013 & 2,1 & 12,33 \\
\hline PA & 2 & 2,7 & 6,6 & 0,013 & 4,3 & 12,47 \\
\hline PSG & 2 & 5,0 & 8,1 & 0,010 & 5,7 & 12,07 \\
\hline OITA & 2 & 4,5 & 9,2 & 0,017 & 3,8 & 12,75 \\
\hline ASC & 1 & 5,2 & 6,5 & 0,048 & 2,4 & 12,80 \\
\hline ASC & 2 & 5,0 & 4,8 & 0,034 & 2,2 & 12,81 \\
\hline GN & 2 & 5,1 & 6,5 & 0,044 & 2,4 & 12,42 \\
\hline JG & 2 & 4,1 & 6,5 & 0,032 & 2,9 & 12,66 \\
\hline $\mathrm{CA}$ & 2 & 5,3 & 6,3 & 0,007 & 3,1 & 12,65 \\
\hline $\mathrm{PF}$ & 2 & 8,8 & 8,0 & 0,010 & 6,8 & 12,42 \\
\hline Média & & 6,6 & 6,5 & 0,010 & 4,0 & \\
\hline \multirow{4}{*}{ Limites de detecção } & Técnica & & & & & \\
\hline & GF-AAS & 0,0020 & & 0,0004 & & \\
\hline & F-AAS (N2O-acet.) & 0,40 & & 0,04 & 0,04 & \\
\hline & F-AAS (ar-acet.) & & 0,03 & 0,00 & & \\
\hline
\end{tabular}


custo (Freitas 2008). Portanto, para cada tonelada de lama residual gerada, temos uma descarga de aproximadamente $440 \mathrm{~L}$ de água pura, considerando-se a densidade $(\rho=1,00 \mathrm{~kg} / \mathrm{L})$ dessa substância nas condições ambientais (Freitas 2008).

Algumas empresas têm utilizado filtro prensa em seu processo produtivo, no sentido de diminuir o teor de água final no rejeito gerado e reutilizar esta água no processo de forma a mitigar seu possível impacto ambiental. Normalmente, esse teor diminui para $30 \%$ ou menos (Prezotti et al., 2004).

Avaliação do pH Com a adição de altos volumes de cal durante o processamento das rochas ornamentais, espera-se elevado $\mathrm{pH}$ no rejeito final, como resultado da dissolução da cal hidratada (Eq. 1 e 2):

$$
\begin{aligned}
& \mathrm{CaO}+\mathrm{H}_{2} \mathrm{O} \rightarrow \mathrm{Ca}(\mathrm{OH})_{2}(1) \\
& \mathrm{Ca}(\mathrm{OH})_{2} \rightarrow \mathrm{Ca}^{2+}+2 \mathrm{OH}^{-}(2)
\end{aligned}
$$

$\mathrm{O} \mathrm{pH}$ aproximado desta reação está mostrado na Eq. 3:

$$
\mathrm{Kps}=\left[\mathrm{Ca}^{2+}\right] \cdot\left[\mathrm{OH}^{-}\right] 2(3)
$$

onde, $\left[\mathrm{Ca}^{2+}\right]=\mathrm{x}\left[\mathrm{OH}^{-}\right]=2 \times$ para $\mathrm{Kps}=$ $5,02 \cdot 10^{-6}($ Hodgman 2008), com $\mathrm{pH}=12,33$.

Na Tab. 3 são apresentadas as medidas de $\mathrm{pH}$ para os diversos rejeitos originais deste trabalho, em função do momento da serragem [início (1), meio (2) e fim (3)], conforme dados apresentados na Tab. 1. Altos valores de $\mathrm{pH}$ foram observados devido à solubilização da cal hidratada (próximo de 12,33). Nota-se, em geral, que o $\mathrm{pH}$ dos rejeitos varia entre as empresas e de acordo com o tipo de bloco serrado, e que ainda para o mesmo bloco não há uma tendência evidente de queda ou aumento no $\mathrm{pH}$, como consequência de uma provável não uniformidade no emprego dos insumos (principalmente cal) (Freitas 2008).

Tal variabilidade pode ser atribuída à falta de uniformidade no processo industrial, no qual não existe um registro adequado da quantidade de insumos durante o processo, que fica dependente da experiência e da capacidade de percepção dos encarregados da serragem (Ribeiro 2005).

É fato que os pHs dos rejeitos eliminados na expurga de cada tear amostrado, em geral, estão muito elevados, devendo ocasionar solubilizações de espécies químicas indesejáveis
Tabela 3 - Valores de $p H$ das amostras originais para início (1), meio (2) e fim (3), da serragem

\begin{tabular}{|c|c|c|c|}
\hline \multirow{2}{*}{ Amostras } & \multirow{2}{*}{ Etapas } & \multicolumn{2}{|c|}{ pH das soluções } \\
\hline & & Original & Média \\
\hline \multirow{3}{*}{ Branco Siena (BS) } & 1 & 12,61 & \multirow{3}{*}{12,52} \\
\hline & 2 & 12,46 & \\
\hline & 3 & 12,50 & \\
\hline \multirow{3}{*}{ Verde Pavão (VP) } & 1 & 12,34 & \multirow{3}{*}{12,24} \\
\hline & 2 & 12,08 & \\
\hline & 3 & 12,29 & \\
\hline \multirow{3}{*}{$\begin{array}{l}\text { Amarelo São Francisco } \\
\text { (ASF) }\end{array}$} & 1 & 12,80 & \multirow{3}{*}{12,69} \\
\hline & 2 & 12,70 & \\
\hline & 3 & 12,57 & \\
\hline \multirow{3}{*}{ Amarelo Icaraí (AI) } & 1 & 12,85 & \multirow{3}{*}{12,8} \\
\hline & 2 & 12,91 & \\
\hline & 3 & 12,64 & \\
\hline \multirow{3}{*}{ Beje Ipanema (BI) } & 1 & 12,54 & \multirow{3}{*}{12,51} \\
\hline & 2 & 12,33 & \\
\hline & 3 & 12,65 & \\
\hline \multirow{3}{*}{ Preto Aracruz (PA) } & 1 & 12,50 & \multirow{3}{*}{12,51} \\
\hline & 2 & 12,47 & \\
\hline & 3 & 12,57 & \\
\hline \multirow{3}{*}{ Preto São Gabriel (PSG) } & 1 & 12,46 & \multirow{3}{*}{12,25} \\
\hline & 2 & 12,07 & \\
\hline & 3 & 12,22 & \\
\hline \multirow{3}{*}{ Ocre Itabira (OITA) } & 1 & 12,68 & \multirow{3}{*}{12,74} \\
\hline & 2 & 12,75 & \\
\hline & 3 & 12,80 & \\
\hline \multirow{3}{*}{ Cinza Corumbá (CC) } & 1 & 12,20 & \multirow{3}{*}{12,28} \\
\hline & 2 & 12,29 & \\
\hline & 3 & 12,34 & \\
\hline \multirow{3}{*}{$\begin{array}{l}\text { Amarelo Santa Cecília } \\
\text { (ASC) }\end{array}$} & 1 & 12,80 & \multirow{3}{*}{12,8} \\
\hline & 2 & 12,81 & \\
\hline & 3 & 12,80 & \\
\hline \multirow{3}{*}{ Giallo Napoleone (GN) } & 1 & 12,48 & \multirow{3}{*}{12,47} \\
\hline & 2 & 12,42 & \\
\hline & 3 & 12,50 & \\
\hline & 1 & 12,70 & \\
\hline Jade Green (JG) & 2 & 12,66 & 12,68 \\
\hline & 3 & 12,67 & \\
\hline & 1 & 12,35 & \\
\hline Cinza Andorinha (CA) & 2 & 12,65 & 12,46 \\
\hline & 3 & 12,39 & \\
\hline & 1 & 12,47 & \\
\hline Preto Florido (PF) & 2 & 12,42 & 12,41 \\
\hline & 3 & 12,35 & \\
\hline & $\begin{array}{l}\text { Média } \\
\text { global }\end{array}$ & 12,53 & \\
\hline
\end{tabular}
dos blocos estudados, acompanhados das médias tipificadas e global 
$(\mathrm{Al} / \mathrm{pH}>6,0$ e $\mathrm{Cr} / \mathrm{pH}>10,0)$, na forma de hidroxicomplexos (Skoog et al. 2006). Essa hipótese é confirmada quando são comparados os teores de cromo total - teor referente à abertura total efetuada em amostras residuais - (Fig. 3) com os de $\mathrm{pH}$ para as amostras de rejeitos, tendo-se obtido boas correlações.

Determinação de metais pela técnica AAS e FAAS No sentido de aprimorar as informações sobre o rejeito original proveniente do corte dos blocos de granito, os teores de alguns metais foram determinados por F AAS após abertura total

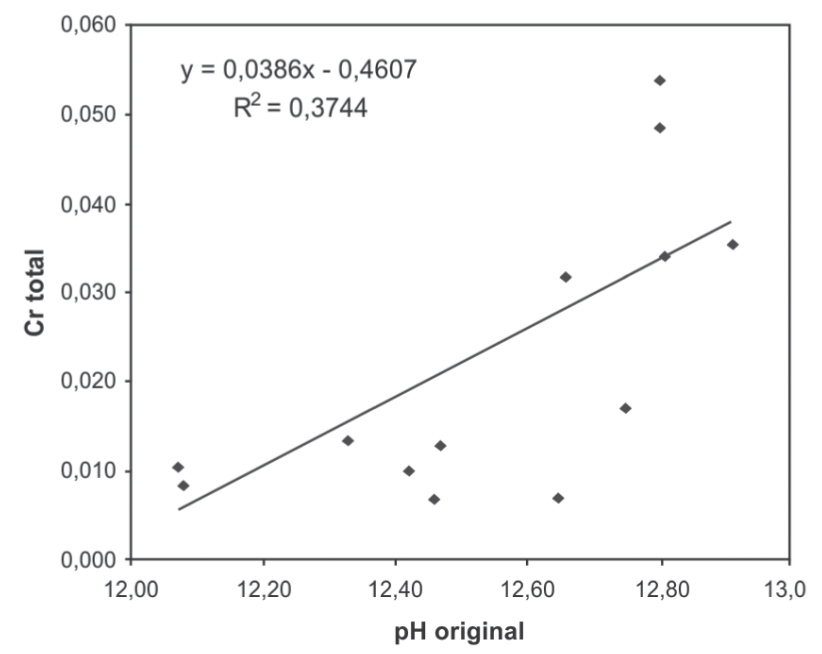

Figura 3 - Correlação entre cromo total e pH das amostras originais de rejeito de granito.

Tabela 4-Correlações de Pearson de pH com teores totais de alguns metais nos rejeitos secos

\begin{tabular}{lccccc}
\hline & & Fe total & Cr total & Ca total & \\
\hline \multirow{4}{*}{ Fe total } & $\mathrm{R}$ & 0,2 & & & \\
& $\mathrm{Sig}$ & 0,492 & & & \\
& $\mathrm{n}$ & 14 & & & \\
\hline \multirow{4}{*}{ Cr total } & $\mathrm{R}$ & $-0,123$ & 0,203 & & \\
& $\mathrm{Sig}$ & 0,676 & 0,487 & & \\
& $\mathrm{~N}$ & 14 & 14 & & \\
\hline \multirow{4}{*}{ Ca total } & $\mathrm{R}$ & 0,389 & $0,696\left(^{* *}\right)$ & $-0,236$ & \\
& $\mathrm{Sig}$ & 0,169 & 0,006 & 0,416 & \\
\hline \multirow{2}{*}{ pH } & $\mathrm{N}$ & 14 & 14 & 14 & \\
original & $\mathrm{R}$ & $-0,19$ & $-0,004$ & $0,617\left(^{*}\right)$ & $-0,304$ \\
& $\mathrm{Sig}$ & 0,515 & 0,99 & 0,019 & 0,291 \\
& $\mathrm{~N}$ & 14 & 14 & 14 & 14 \\
\hline
\end{tabular}

*correlação é significante para o nível de 0,05 (teste bicaudal); **correlação para o nível de 0,01 (teste bicaudal); R: coeficiente de Pearson; Sig: significância; n: população total analisada. das amostras secas em estufa $\left(42{ }^{\circ} \mathrm{C}\right)$, com uma combinação de ácidos e peróxido de hidrogênio. Esses resultados são apresentados na Tab. 2 .

Há uma razoável correlação observada entre $\mathrm{Fe}$ e $\mathrm{Ca}$ total nos rejeitos (Tab. 4), confirmando a introdução combinada destes dois insumos durante o processo de serragem dos blocos de granito, visando à redução da corrosividade das lâminas e da própria granalha. Correlação significativa também foi observada para $\mathrm{Cr}$ total e $\mathrm{pH}$ original, conforme supracitado.

Contudo, não foram observadas correlações entre alumínio e cálcio totais em relação à composição total dessas duas espécies químicas presentes no resíduo, já que os mesmos são componentes dos minerais majoritários presentes nas rochas silicáticas e no insumo utilizado na manufatura das mesmas, respectivamente, em estudo (por exemplo, feldspatos plagioclásios).

CONCLUSÕES Neste trabalho foi possível coletar diversos tipos de amostras de resíduos de serragem de granitos e de mármores, de empresas diferentes e distintas regiões geológicas, o que aponta para uma possibilidade de avaliação da uniformidade no processo de beneficiamento das rochas ornamentais.

É fundamental que sejam associados parâmetros tecnológicos tradicionais de rochas com outros existentes, não utilizados neste setor produtivo, como o monitoramento do $\mathrm{pH}$ dos rejeitos e de teores metálicos. Mais informações tendem a facilitar o processo de gerenciamento da geração e destinação dos resíduos de serragem da indústria de rochas ornamentais.

Com média de $44,3 \%$ e desvio padrão de $13 \%$, o setor de rochas ornamentais parece apresentar coerência pelo menos no consumo de água no processo de serragem, embora este teor sugira uma demanda altíssima de água no beneficiamento de rochas.

Apesar da relativa uniformidade do volume consumido de água nos processos de serragem de rochas, este recurso ainda é utilizado de forma pouco sustentável, principalmente por empresas de médio e pequeno porte. O elevado custo de novas tecnologias, como o filtro prensa, e o fato da redução notável da alcalinidade do resíduo, devido à formação de carbonatos $\left(\mathrm{CO}_{3}{ }^{2-}\right)$, 
quando este é disposto em grandes superfícies de contato e condições de temperatura elevada, podem dirigir o setor a tomar medidas mais econômicas e eficazes em curto prazo, em busca da gestão adequada do recurso hídrico. Nesse sentido, os leitos de secagem parecem representar uma boa alternativa. Os leitos de secagem são tanques feitos em superfícies planas, com declive para um dos lados, com drenagem padrão, em que resíduos ou lamas de serragem podem ser dispostos ao ar livre e em grande superfície de contato com o ar atmosférico.

Levando-se em conta a NBR 10.004 (ABNT 2004a), algumas das amostras de resíduos analisadas apresentaram pHs maiores do que 12,5, o que já é suficiente para caracterizá-los como resíduos perigosos (Classe I) e, portanto, corrosivos.

A avaliação do $\mathrm{pH}$ nos diferentes momentos da serragem de blocos graníticos revelou extrema heterogeneidade dos processos industriais amostrados, mesmo para materiais de uma mesma empresa e, em geral, não foram detectadas tendências de elevação ou decréscimo de alcalinidade nos resíduos. Como este parâmetro está intimamente ligado à cal utilizada no referido processo (demonstrado por meio de estudos de correlação), fica evidente que, neste quesito, as empresas adotam critérios de difícil análise científica. A impressão é de que as medidas de viscosidade da lama, normalmente realizadas durante o processo de serragem, não são suficientes para avaliar tendências químicas no rejeito gerado.

A inserção de cal no processo de serragem dos blocos rochosos está diretamente ligada à alta basicidade do resíduo.

Finalmente, o resíduo de corte de mármores, aparentemente, é muito menos agressivo do que o de granitos comerciais (rochas silicáticas), devido ao menor teor de cal e granalha utilizados na serragem. Contudo, havendo uma simples segregação entre resíduos de rochas silicáticas e carbonáticas, certamente o valor agregado desses resíduos e suas potencialidades seriam majoradas.

AGRADECIMENTOS Os autores agradecem ao Serviço Brasileiro de Apoio às Micro e Pequenas Empresas (SEBRAE) e ao Sindirochas pelo apoio financeiro, ao Programa de PósGraduação em Química da UFES pelo apoio institucional e ao Fundo de Apoio à Ciência e Tecnologia (FACITEC - Prefeitura Municipal de Vitória - ES) pelo financiamento de bolsas de pesquisa em níveis de Mestrado e iniciação científica.

\section{References}

ASSOCIAÇÃO BRASILEIRA DE NORMAS TÉCNICAS. 2004a. NBR 10.004: Resíduos sólidos: classificação, Rio de Janeiro.

ASSOCIAÇÃO BRASILEIRA DE NORMAS TÉCNICAS. 2004b. NBR 10.006: Procedimento para obtenção de extrato solubilizado de resíduos sólidos, Rio de Janeiro.

ASSOCIAÇÃO BRASILEIRA DE NORMAS TÉCNICAS. 2004c. NBR 10.007: Amostragem de resíduos sólidos, Rio de Janeiro.

Freitas J.J. 2008. Caracterização química inorgânica de rejeitos segregados de serragem de rochas ornamentais do Espírito Santo. Dissertação de Mestrado, Programa de Pós-Graduação em Química, Centro de Ciências Exatas, Universidade Federal do Espírito Santo, Vitória, $113 \mathrm{p}$.

Hodgman C.D. 2008. CRC Handbook of Chemistry and Physics. 88. ed., New York, CRC /Taylor e Francis Press.
Peyneau G.O.R. \& Pereira G.S. 2004. Tratamento dos resíduos provenientes das serragens de granito através da implantação do filtro-prensa. In: XXIV ENEGEP, p. 1-5, Florianópolis. Disponível em: www.uvv.com.br. Acessado em: 05/05/2008.

Prezotti J.C.S., Braga F.S., Couto M.C.L., Batista T.R.O., Yamane L.H. 2004. Proposta de implantação de uma central de tratamento de resíduos do beneficiamento de rochas ornamentais em Nova Venécia - ES. Disponível em: http://www.manancialprojetos.com.br/ port/t_artigo.asp. Acessado em: 17/06/2008.

Ribeiro R.P. 2005. Influência das características petrográfica de granitos no processo industrial de desdobramento de blocos. Tese de Doutorado, Programa de Pós-Graduação da Escola de Engenharia de São Carlos, Departamento de Geotecnia, Universidade Federal de São Carlos, São Carlos, $132 \mathrm{p}$. 
Sampaio J.A., França S.C.A., Braga P.F.A. 2007. Tratamento de minérios: práticas laboratoriais. Rio de Janeiro, CETEM/MCT.

SEMACE - Superintendência Estadual de Meio Ambiente do Ceará. 2005. Identificação de metais nos sedimentos dos estuários da costa do estado. Disponível em: http://www.semace.ce.gov.br/programas/zee/Produtos/ MetaisPesado.pdf. Acessado em: 07/04/2008.

Skoog D., West D.M., Holler D.M. III., Crouch S.R. 2006. Fundamentos de química analítica. 8. ed. São Paulo, Pioneira Thomson Learning, 21:553-576.
Vieira Júnior H.T. 2001. Proposta de recuperação da granalha não ativa no desdobramento de rochas ornamentais em teares multilâminas. Dissertação de Mestrado, Departamento de Metalurgia da Escola de Engenharia, Programa de Pós-Graduação em Engenharia de Minas, Metalúrgica e de Materiais, Universidade Federal do Rio Grande do Sul, Porto Alegre, $67 \mathrm{p}$.

Manuscrito ID 18080

Recebido em: 22/07/2010

Aprovado em: 23/04/2012 